\title{
Composición Factorial de la Escala de Ansiedad ante el Envejecimiento de Lasher y Faulkender en Estudiantes Universitarios Mexicanos
}

\author{
Martha Ornelas $^{(1)}$, Gabriel Gastélum ${ }^{(1)}$, Jeanette Lopez-Walle ${ }^{(2)}$ y Judith M. Rodríguez-Villalobos ${ }^{(1) \star ~}$ \\ (1)Universidad Autónoma de Chihuahua. Facultad de Ciencias de la Cultura Física. DES Salud CA 1121. \\ Calle Escorza 900, CP 31000 Chihuahua, Chih.- México. (e-mail: mornelas@uach.mx, \\ gastelum@uach.mx, jeanette.lopezw@uanl.mx y jurodrig@uach.mx) \\ (2)Universidad Autónoma de Nuevo León. Facultad de Organización Deportiva. Cd. Universitaria, s/n, \\ San Nicolás de los Garza, N. L., México (e-mail: jeanette.lopezw@uanl.mx) \\ * autor a quien debe ser dirigida la correspondencia
}

Recibido Jul. 30, 2015; Aceptado Sep. 29, 2015; Versión final Oct. 15, 2015, Publicado Abr. 2016

\begin{abstract}
Resumen
En el presente estudio se analiza si es posible replicar los resultados psicométricos propuestos por Lasher y Faulkender para la Escala de Ansiedad ante el Envejecimiento, en un grupo de estudiantes universitarios mexicanos. La muestra total fue de 825 participantes con una edad media de aproximadamente 20.8 años. Toso eran estudiantes varones de las licenciaturas de Educación Física y Motricidad Humana que se ofrecen en la Universidad Autónoma de Chihuahua en México. La estructura factorial del cuestionario se analizó a través de análisis factoriales confirmatorios. Los análisis, muestran que una estructura tetra factorial es adecuada ya que a través de criterios estadísticos y sustantivos, ha mostrado adecuados indicadores de fiabilidad y validez. Los cuatro factores son: (i) miedo a las personas mayores; (ii) apariencia física; (iii) miedo a las pérdidas; y (iv) preocupaciones psicológicas. Además, los resultados de los análisis factoriales llevados a cabo con las submuestras, indican la existencia de fuertes evidencias de la estabilidad de la estructura factorial.
\end{abstract}

\section{Factorial Structure of the Anxiety Aging Scale of Lasher and Faulkender in Mexican University Students}

\begin{abstract}
In the present study the possibility of replicate the psychometric results proposed by Lasher and Faulkender for Anxiety Aging Scale, in a group of Mexican university students. The total sample comprised 825 participants with a mean age of 20.8 years. All of them were male students of the majors in Physical Education and in Human Motricity offered at the Autonomous University of Chihuahua in Mexico. The factorial structure of the questionnaire was analyzed using confirmatory factor analysis. The psychometric analysis showed that a tetra-factorial structure was adequate since through statistic criteria it showed adequate indicators of reliability and validity. The four factors were: (i) fear of old people; (ii) physical appearance; (iii) fear of losses; and (iv) psychological concerns. In addition to this, factor analysis conducted with subsamples indicated the presence of strong evidence of factor structure stability.
\end{abstract}

Keywords: anxiety, factorial structure, construct validation, structural equation 


\section{INTRODUCCIÓN}

En una sociedad como la nuestra dominada por factores económicos, en la que ser productivo es un elemento fundamental, con demasiada frecuencia el valor de las personas está condicionado a su capacidad para producir da origen a que los adultos mayores tengan un papel marginal quedando en buena medida excluidos de la propia dinámica social y dado que la proporción de adultos mayores continúa incrementándose, se torna cada vez más importante comprender los determinantes del comportamiento discriminatorio y de los estereotipos contra las personas de la tercera edad (actitudes ageístas), especialmente entre los estudiantes universitarios, con el fin de promover actitudes más positivas y aumentar el interés en el estudio sobre el envejecimiento y la atención y trabajo para con el adulto mayor.

La ansiedad sobre el envejecimiento se puede definir como la preocupación y anticipación de las pérdidas físicas, mentales y personales adversas durante el proceso de envejecimiento (Lasher y Faulkender, 1993); ansiedad que influye en las actitudes y comportamientos hacia los ancianos (Allan y Johnson, 2008; Boswell, 2012) y a la adaptación al propio proceso de envejecimiento (Bousfield y Hutchison, 2010). Esta investigación se basa pues en la premisa de que la ansiedad ante el envejecimiento es un importante factor, mediador en las actitudes y comportamientos hacia las personas de edad avanzada, así como en la adaptación a los propios procesos de envejecimiento. Se propone un modelo multidimensional de ansiedad ante el envejecimiento y se evalúa la validez factorial del modelo.

El presente estudio instrumental (Montero y León, 2005) se ha dirigido a proporcionar apoyo empírico a la división factorial de la Escala de Ansiedad ante el Envejecimiento de Lasher y Faulkender (1993); lo que se justifica por la importancia de comprobar la estructura factorial de un instrumento y la equivalencia psicométrica del mismo en distintos grupos; ya que en el contexto de la comparación intergrupal, es indispensable plantearse la necesidad de llevar a cabo la adaptación de un instrumento de medida psicológica que cumpla con todos los criterios de equivalencia, pero sobre todo plantearse si la misma estructura factorial es aplicable a distintos grupos de sujetos o, de modo más genérico, a distintas poblaciones (Abalo et al., 2006).

Por tanto, este trabajo pretende, por una parte, indagar si se replican los resultados psicométricos propuestos por Lasher y Faulkender para la Escala de Ansiedad ante el Envejecimiento y, por otra, ampliarlos. Para ello, en primer lugar se comprobará el grado de congruencia de la estructura factorial de dicho cuestionario obtenida en el presente estudio y la reportada por Lasher y Faulkender (1993). En segundo lugar, se calculará la invarianza factorial entre las submuestras del presente estudio.

\section{METODOLOGÍA}

\section{Participantes}

La muestra de 825 participantes se obtuvo mediante un muestreo por conveniencia, tratando de abarcar la representatividad de las diferentes licenciaturas que se ofrecen en la Facultad de Ciencias de la Cultura Física de la Universidad Autónoma de Chihuahua. La edad de los participantes fluctuó entre los 18 y 26 años, con una media de 20.77 y una desviación estándar de 1.83 años. La muestra fue aleatoriamente dividida en dos partes utilizando el Statistical Package for the Social Sciences (SPSS) en su versión 18.0; con el fin de realizar estudios paralelos que permitieran corroborar y verificar los resultados obtenidos (validación cruzada). La submuestra 1 quedo constituida por 414 hombres. Las edades fluctúan entre los 18 y 26 años, con una media de 20.79 y una desviación estándar de 1.82 años. La submuestra 2 quedo compuesta por 411 hombres. Las edades fluctúan entre los 18 y 26 años, con una media de 20.75 y una desviación estándar de 1.84 años.

\section{Instrumento}

Escala de Ansiedad ante el Envejecimiento de Lasher y Faulkender versión en español de Rivera et al. (2007). Cuestionario tipo Likert que consta de 20 ítems que se agrupan en cuatro dimensiones o subescalas: Miedo a las Personas Mayores, Apariencia Física, Miedo a las Pérdidas y Preocupaciones Psicológicas; donde el encuestado responde, en una escala de 0 a 10 su grado de acuerdo con cada uno de los aspectos propuestos.

\section{Procedimiento}

Se invitó a participar en el estudio a los alumnos de las licenciaturas que se ofrecen en la Facultad de Ciencias de la Cultura Física (FCCF) de la Universidad Autónoma de Chihuahua. Los que aceptaron participar firmaron la carta de aceptación correspondiente. Luego se aplicó el instrumento, antes descrito, por medio de una computadora personal (módulo administrador del instrumento del editor de escalas de 
ejecución típica), en una sesión de aproximadamente 30 minutos; en los laboratorios o centros de cómputo de la FCCF. Al inicio de cada sesión se hizo una pequeña introducción sobre la importancia de la investigación y de cómo acceder al instrumento. Se les solicitó la máxima sinceridad y se les garantizó la confidencialidad de los datos que se obtuvieran. Las instrucciones de cómo responder se encontraban en las primeras pantallas; antes del primer reactivo del instrumento. Al término de la sesión se les agradeció su participación. Una vez aplicado el instrumento se procedió a recopilar los resultados por medio del módulo generador de resultados del editor de escalas versión 2.0 (Blanco et al., 2013).

\section{Análisis de datos}

El primer paso del análisis de las propiedades psicométricas del cuestionario consistió en calcular la media, la desviaciones estándar, la asimetría, la curtosis y los índices de discriminación de cada ítem. Para luego eliminar de la escala aquellos que obtienen una curtosis o asimetría extremas, o un índice de discriminación por debajo de 0.30. Luego, se sometieron a comparación dos modelos de medida: el Modelo 1 (M1), modelo de cuatro factores acorde a la distribución original de los ítems dentro del cuestionario y el Modelo 2 (M1b), que responde a la estructura factorial del modelo anterior, eliminando los ítems que no fueron suficientemente bien explicados por ese modelo y/o obtuvieron un índice de discriminación bajo.

Para conducir los análisis factoriales confirmatorios se utilizó el software AMOS 21 (Arbuckle, 2012), las varianzas de los términos de error fueron especificados como parámetros libres, en cada variable latente (factor) se fijó uno de los coeficientes estructurales asociados a uno, para que su escala sea igual a la de una de las variables observables (ítems). El método de estimación empleado fue el de Máxima Verosimilitud; siguiendo la recomendación de Thompson (2004), en el sentido de que cuando se emplea análisis factorial confirmatorio se debe corroborar no sólo el ajuste de un modelo teórico sino que es recomendable comparar los índices de ajuste de varios modelos alternativos para seleccionar el mejor.

Para evaluar el ajuste del modelo se emplearon el estadístico Chi-cuadrado, el índice de bondad de ajuste (GFI) y el error cuadrático medio de aproximación (RMSEA) como medidas absolutas de ajuste. El índice de bondad ajustado (AGFI), el Índice Tucker-Lewis (TLI) y el índice de bondad de ajuste comparativo (CFI) como medidas de ajuste incremental. La razón de Chi-cuadrado sobre los grados de libertad (CMIN/GL) y el Criterio de Información de Akaike (AIC) como medidas de ajuste de parsimonia (Byrne, 2010; Gelabert et al., 2011). Donde valores de .90 a menos de .95 para GFI, AGFI y CFI y de .8 a .1 para RMSEA corresponden a un ajuste aceptable y mayores o iguales a .95 para GFI, AGFI y CFI y menores de .8 para RMSEA a un ajuste óptimo (Hooper et al., 2008). Siguiendo las recomendaciones de Abalo et al. (2006), se llevó a cabo un análisis de la invarianza factorial del cuestionario para las submuestras tomando como base el mejor modelo de medida obtenido en la etapa anterior. Por último se calculó la fiabilidad de cada una de las dimensiones, de los modelos de medida obtenidos en cada submuestra, a través del Coeficiente Alpha de Cronbach (Nunnally y Bernstein, 1995) y del Coeficiente Omega (Revelle y Zinbarg, 2009; Sijtsma, 2009); donde valores $>.9$ se consideran excelente consistencia interna, $>.8$ buena y $>.7$ aceptable (Darren y Mallery, 2013).

\section{RESULTADOS}

\section{Análisis descriptivos e índices de discriminación}

En la Tabla 1 se resumen los resultados de los análisis descriptivos y los índices de discriminación (correlación elemento-total corregida) de cada uno de los 20 ítems del cuestionario en la muestra total. Las respuestas a todos los ítems reflejan unas puntuaciones medias que oscilan entre 1.27 y 7.74 , y la desviación estándar ofrece, en todos los casos, valores mayores a 1.60 (dentro de un rango de respuesta entre 0 y 10). Con excepción de los reactivos Me gusta visitar a mis familiares viejos (3) y He mentido acerca de mi edad a fin de parecer más joven (4) todos los valores de asimetría y curtosis se encuentran dentro del rango \pm 2.5 ; por lo que se infiere que las variables se ajustan razonablemente a una distribución normal. En cuanto a los índices de discriminación la mayoría de los ítems discriminan satisfactoriamente; únicamente los ítems 6 (De cuando llegue a ser viejo, es mi salud lo que más me preocupa), 7 (Tendré mucho en que ocupar mi tiempo cuando sea viejo) y 16 (Creo que cuando sea viejo todavía podré hacer casi todas las cosas por mí mismo) obtienen un índice de discriminación por debajo de .30 (Brzoska y Razum, 2010).

\section{Análisis factoriales confirmatorios}

Los resultados globales del análisis factorial confirmatorio en la submuestra 1 (GFI .908; RMSEA .064; CFI .910) y la submuestra 2 (GFI .890; RMSEA .073; CFI .890) para el modelo M1 que corresponde a la distribución original de los ítems dentro del cuestionario de la Escala de Ansiedad ante el Envejecimiento de Lasher y Faulkender, indican que el modelo de medición, en ambas submuestras se puede considerar como aceptable aunque no óptimo (Tabla 2). En esta tabla, ${ }^{*} p<.05 ; \mathrm{GFI}=$ índice de bondad de ajuste; RMSEA = 
raíz del error medio; AGFI = índice corregido de la bondad de ajuste; TLI = índice de Tucker-Lewis; CFI = índice de ajuste comparativo; CMIN/DF = índice de ajuste chi cuadrado dividido por los grados de libertad; $\mathrm{AIC}=$ criterio de información de Akaike

Tabla 1: Análisis descriptivos e índices de discriminación de los ítems del cuestionario "Escala de Ansiedad ante el Envejecimiento". Muestra total. $\mathrm{M}$ = media; $\mathrm{DE}$ = desviación estándar; $\mathrm{AS}$ = asimetría; $\mathrm{CU}=$ curtosis; $r_{\text {i-total }}=$ correlación elemento-total corregida .

\begin{tabular}{cccccc}
\hline Ítem & $\mathrm{M}$ & $\mathrm{DE}$ & $\mathrm{AS}$ & $\mathrm{CU}$ & $\mathrm{r}_{\text {i-total }}$ \\
\hline Item 1 & 2.19 & 1.73 & .78 & .82 & .38 \\
Item 2 & 5.28 & 3.16 & -.28 & -1.02 & .30 \\
Item 3 & 1.84 & 1.89 & 1.38 & 2.59 & .33 \\
Item 4 & 1.27 & 2.47 & 2.12 & 3.56 & .34 \\
Item 5 & 3.05 & 3.13 & .71 & -.69 & .54 \\
Item 6 & 7.74 & 2.40 & -1.38 & 1.82 & .09 \\
Item 7 & 3.24 & 2.10 & .41 & .08 & .22 \\
Item 8 & 5.55 & 3.05 & -.43 & -.82 & .52 \\
Item 9 & 3.13 & 3.04 & .59 & -.84 & .63 \\
Item 10 & 1.80 & 1.81 & 1.25 & 2.16 & .45 \\
Item 11 & 2.00 & 1.87 & 1.09 & 1.60 & .53 \\
Item 12 & 3.46 & 3.24 & .52 & -1.00 & .61 \\
Item 13 & 2.20 & 1.83 & .70 & .38 & .39 \\
Item 14 & 5.55 & 3.13 & -.37 & -.93 & .51 \\
Item 15 & 3.64 & 3.19 & .40 & -1.06 & .70 \\
Item 16 & 2.86 & 1.99 & .71 & .71 & .29 \\
Item 17 & 4.60 & 3.14 & -.03 & -1.17 & .57 \\
Item 18 & 2.15 & 1.87 & .95 & 1.26 & .49 \\
Item 19 & 1.56 & 1.64 & 1.09 & 1.35 & .41 \\
Item 20 & 3.26 & 3.05 & .51 & -.94 & .60 \\
\hline
\end{tabular}

Tabla 2: Índices absolutos, incrementales y de parsimonia para los modelos generados. Submuestras 1 y 2.

\begin{tabular}{|c|c|c|c|c|c|c|c|c|}
\hline \multirow[b]{2}{*}{ Modelo } & \multicolumn{3}{|c|}{ Índices absolutos } & \multicolumn{3}{|c|}{ Índices incrementales } & \multicolumn{2}{|c|}{ Índices de parsimonia } \\
\hline & $\chi^{2}$ & GFI & RMSEA & AGFI & TLI & $\mathrm{CFI}$ & CMIN/DF & AIC \\
\hline \multicolumn{9}{|c|}{ Primera solución factorial (submuestra 1) } \\
\hline M1 & $438.453^{*}$ & .908 & .064 & .882 & .895 & .910 & 2.673 & 530.453 \\
\hline M1b & $126.749^{*}$ & .956 & .044 & .936 & .971 & .978 & 1.785 & 194.749 \\
\hline \multicolumn{9}{|c|}{ Segunda solución factorial (submuestra 2) } \\
\hline M1 & $522.224^{*}$ & .890 & .073 & .859 & .872 & .890 & 3.184 & 614.224 \\
\hline M1b & $190.648^{*}$ & .941 & .064 & .912 & .941 & .954 & 2.685 & 258.648 \\
\hline
\end{tabular}

El conjunto de los cuatro factores del modelo M1 explican aproximadamente el $57 \%$ de la varianza en la primer submuestra y el $59 \%$ de la varianza en la segunda submuestra. Por otro lado siete de los 20 ítems, en ambas submuestras, saturan por debajo de .70 en su dimensión prevista (2 temo que cuando sea viejo todos mis amigos se hayan muerto, 3 me gusta visitar a mis familiares viejos, 4 he mentido acerca de mi edad a fin de parecer más joven, 5 creo que será muy difícil para mí el sentirme contento cuando sea viejo, 6 de cuando llegue a ser viejo, es mi salud lo que más me preocupa, 7 tendré mucho en que ocupar mi tiempo cuando sea viejo y 16 creo que cuando sea viejo todavía podré hacer casi todas las cosas por mí mismo). Observándose además, intercorrelaciones de bajas a moderadas entre los cuatro factores evidenciando una adecuada validez discriminante entre ellos.

Los resultados globales del análisis factorial confirmatorio en la primer (GFI .956; RMSEA .044; CFI .978) y segunda submuestra (GFI .958; RMSEA .044; CFI .980), del segundo modelo sometido a prueba (M1b) que corresponde a una estructura tetradimensional del cuestionario sin los ítems de más baja saturación en 
cada uno de los factores, indican que este modelo de medición es mejor que el modelo anterior y que su ajuste es óptimo (Tabla 2). Los cuatro factores de este modelo explican en conjunto, en ambas submuestras aproximadamente el $68 \%$ de la varianza.

Por otro lado de acuerdo a los resultados de la Tabla 3; solo tres de los 14 ítems, en ambas submuestras, saturan por debajo de .70 en su dimensión prevista. Observándose además, intercorrelaciones de bajas a moderadas entre los cuatro factores evidenciando una adecuada validez discriminante entre ellos.

Tabla 3 Soluciones estandarizadas análisis factorial confirmatorio para el Modelo M1b. Submuestra 1 y 2 . Nota: F1 = Miedo a las personas mayores, F2 = Apariencia física, F3 = Miedo a las pérdidas, 4 = Preocupaciones psicológicas

\begin{tabular}{|c|c|c|c|c|c|c|c|c|}
\hline \multirow[b]{2}{*}{ Item } & \multicolumn{4}{|c|}{ Submuestra 1} & \multicolumn{4}{|c|}{ Submuestra 2} \\
\hline & F1 & F2 & F3 & F4 & $\mathrm{F} 1$ & F2 & F3 & F4 \\
\hline \multicolumn{9}{|l|}{ Pesos Factoriales } \\
\hline 1 Disfruto el estar con gente vieja & .73 & & & & .67 & & & \\
\hline 3 Me gusta visitar a mis familiares viejos & .63 & & & & .65 & & & \\
\hline 10 Disfruto platicar con gente vieja & .81 & & & & .84 & & & \\
\hline $\begin{array}{l}13 \text { Me siento muy a gusto cuando estoy cerca de una } \\
\text { persona vieja }\end{array}$ & .84 & & & & .81 & & & \\
\hline 19 Disfruto hacer cosas por los viejos & .75 & & & & .74 & & & \\
\hline $9 \mathrm{Me}$ molesta imaginarme siendo viejo & & .71 & & & & .70 & & \\
\hline $\begin{array}{l}12 \text { Me ha preocupado el día en que al verme en el espejo } \\
\text { me vea cabellos grises }\end{array}$ & & .74 & & & & .75 & & \\
\hline 15 Verme viejo me ha preocupado & & .83 & & & & .86 & & \\
\hline $\begin{array}{l}20 \text { Cuando me veo en el espejo me molesta ver cómo mi } \\
\text { apariencia ha cambiado con la edad }\end{array}$ & & .67 & & & & .72 & & \\
\hline $\begin{array}{l}8 \text { Me pongo nervioso cuando pienso que alguien tomará } \\
\text { decisiones por mí cuando sea viejo }\end{array}$ & & & 68 & & & & .63 & \\
\hline 14 Me preocupa que la gente me ignore cuando sea viejo & & & .75 & & & & .74 & \\
\hline $\begin{array}{l}17 \text { Me preocupa que la vida pierda sentido para mí cuando } \\
\text { sea viejo }\end{array}$ & & & .70 & & & & .74 & \\
\hline $\begin{array}{l}11 \text { Cuando sea viejo creo que voy a sentirme bien con la } \\
\text { vida }\end{array}$ & & & & .86 & & & & .80 \\
\hline $\begin{array}{l}18 \text { Cuando sea viejo, confío en que me sentiré bien } \\
\text { conmigo mismo }\end{array}$ & & & & .61 & & & & .70 \\
\hline \multicolumn{9}{|l|}{ Correlaciones Factoriales } \\
\hline $\mathrm{F} 1$ & - & & & & - & & & \\
\hline $\mathrm{F} 2$ & .20 & - & & & .26 & - & & \\
\hline F3 & .00 & .80 & - & & .00 & .81 & - & \\
\hline F4 & .65 & .43 & .21 & - & .71 & .51 & .23 & - \\
\hline
\end{tabular}

Invarianza de la estructura factorial entre las submuestras

Los índices de ajuste obtenidos (Tabla 4) permiten aceptar la equivalencia de los modelos de medida básicos entre las dos submuestras. Aunque el valor de Chi-cuadrado excede al exigido para aceptar la hipótesis de invarianza, los índices $\mathrm{GFI}=.948, \mathrm{CFI}=.965$, RMSEA=.039 y AIC=453.397 contradicen esta conclusión lo que nos permite aceptar el modelo base de la invarianza (modelo sin restricciones).

Añadiendo al modelo base restricciones sobre las cargas factoriales caracterizamos la invarianza métrica. Los valores que se recogen en la tabla 4 permiten aceptar este nivel de invarianza. El índice de ajuste general (GFI .966) y el error cuadrático medio de aproximación (RMSEA .037) siguen aportando información convergente en esta dirección. Además, el criterio de información de Akaike (AIC 441.283) y el índice comparativo de Bentler (CFI .966) no sufren grandes variaciones respecto al modelo anterior. Haciendo uso del criterio para la evaluación de los modelos anidados propuesto por Cheung y Rensvold (2002), quiénes sugieren que si el cálculo de la diferencia de los CFI de ambos modelos anidados disminuye en .01 o menos, se da por bueno el modelo restringido y por tanto el cumplimiento de la invarianza factorial; la diferencia entre CFIs obtenida permite aceptar el modelo de invarianza métrica. Podemos concluir hasta ahora que las cargas factoriales son equivalentes en las dos submuestras. 
Una vez demostrada la invarianza métrica entre las submuestras, pasamos a evaluar la equivalencia entre interceptos (invarianza factorial fuerte). Los índices (Tabla 4) muestran un ajuste aceptable de este modelo, tanto evaluado de modo independiente como analizándolo respecto a su anidamiento con el modelo de invarianza métrica. La diferencia entre los índices comparativos de Bentler es de .001; el índice de ajuste general es .946 y el error cuadrático medio de aproximación es .035 Aceptada la invarianza fuerte, los dos modelos evaluados son equivalentes respecto a los coeficientes factoriales y a los interceptos.

Tabla 4: Índices de bondad de ajuste de cada uno de los modelos puestos a prueba en la invarianza factorial. * $p$ $<.05 ; \mathrm{GFI}=$ índice de bondad de ajuste; NFI = índice de ajuste normado; CFI = índice de ajuste comparativo; RMSEA = raíz del error medio; AIC = criterio de Información de Akaike

\begin{tabular}{lccccccc}
\hline \multicolumn{1}{c}{ Modelo } & \multicolumn{7}{c}{ Índice de Ajuste } \\
\cline { 2 - 8 } & $\chi^{2}$ & gl & GFI & NFI & CFI & RMSEA & AIC \\
\hline Modelo sin restricciones & $317.397^{*}$ & 142 & .948 & .939 & .965 & .039 & 453.397 \\
Invarianza métrica & $325.283^{*}$ & 152 & .947 & .938 & .966 & .037 & 441.283 \\
Invarianza factorial fuerte & $327.721^{*}$ & 161 & .946 & .938 & .967 & .035 & 425.721 \\
\hline
\end{tabular}

Los factores obtenidos en los análisis factoriales confirmatorios alcanzan, en su mayoría valores de consistencia interna por encima de 0.75 en ambas submuestras; evidenciando una consistencia interna adecuada para este tipo de subescalas, particularmente si se considera el número reducido de ítems (Tabla 5).

Tabla 5: Coeficiente omega y alfa para los factores obtenidos en los análisis factoriales confirmatorios submuestras 1 y 2.

\begin{tabular}{lllll}
\hline & \multicolumn{2}{c}{ Submuestra 1 } & \multicolumn{2}{c}{ Submuestra 2 } \\
\cline { 2 - 5 } \multicolumn{1}{c}{ Factor } & $\Omega$ & $\alpha$ & $\Omega$ & $\alpha$ \\
\hline Miedo a las personas mayores & .87 &. .86 & .86 & .85 \\
Apariencia física & .83 & .83 & .84 & .84 \\
Miedo a las pérdidas & .75 & .75 & .75 & .74 \\
Preocupaciones psicológicas & .71 & .70 & .72 & .72 \\
\hline
\end{tabular}

\section{DISCUSIÓN}

El objetivo principal del estudio fue indagar si se replican o no los resultados psicométricos propuestos por Lasher y Faulkender (1993) para la Escala de Ansiedad ante el Envejecimiento a través de una muestra de universitarios utilizando el análisis factorial confirmatorio (AFC). Los análisis factoriales confirmatorios realizados en cada submuestra por separado apoyan la estructura factorial de cuatro factores: miedo a las personas mayores, preocupaciones psicológicas, apariencia física y miedo a las pérdidas obtenida por Lasher y Faulkender (1993) al evidenciar una consistencia interna adecuada, particularmente si se considera el número reducido de ítems en cada uno de ellos; al mismo tiempo que los factores así obtenidos presentan en general saturaciones factoriales estandarizadas adecuadas, saturaciones que se corresponden con las halladas en el estudio de Lasher y Faulkender (1993). Sugiriendo además la existencia de fuertes evidencias de la validación cruzada de la medida y por tanto de la estabilidad de la estructura hasta que no se demuestre lo contrario.

En síntesis, el análisis de las propiedades psicométricas de la Escala de Ansiedad ante el Envejecimiento, ha mostrado, tanto en este estudio como en el llevado a cabo por Lasher y Faulkender, que una estructura tetrafactorial es adecuada de acuerdo a los requisitos psicométricos establecidos cuando los informantes son los propios alumnos. La estructura de cuatro factores, atendiendo a criterios estadísticos y sustantivos, ha mostrado adecuados indicadores de ajuste, de fiabilidad y de validez. Sin embargo, el alcance de estos resultados es limitado, y es necesario que en investigaciones futuras se confirme la estructura obtenida, lo cual permitirá contar con evidencia más robusta respecto a la estructura factorial de la escala. Específicamente, debe demostrarse si la invarianza de la estructura de la escala se cumple por género, edad, entre alumnos de distintas licenciaturas, entre otras. Además, resulta inminente la necesidad de realizar estudios transculturales dirigidos a valorar si la estructura factorial de la escala es reflejo o no de diferencias culturales, de tal manera que, se considera que más estudios son necesarios con el fin de 
corroborar o refutar los datos obtenidos en las investigaciones realizadas hasta el momento. Asimismo, es indispensable comprobar si la escala resulta útil para estudiar la relación entre ansiedad ante el envejecimiento y variables tales como el prejuicio y la actitud hacia los adultos mayores.

\section{CONCLUSIONES}

Con base en el estudio presentado, se pueden extraer las siguientes con conclusiones principales:

i) El Análisis Factorial Confirmatorio, en ambas muestras, indicó que el ajuste de los datos al modelo teórico de 14 ítems agrupados en cuatro factores es aceptable.

ii) Los factores en ambas muestras evidenciaron una consistencia interna adecuada, particularmente si se considera el número reducido de ítems en cada uno de ellos

iii) Los resultados del análisis de la invarianza factorial entre las muestras; indican una alta congruencia entre pares de factores.

\section{REFERENCIAS}

Abalo J., Lévy J., Rial A. y Varela J., Invarianza factorial con muestras múltiples, En Modelización con Estructuras de Covarianzas en Ciencias Sociales por J. Lévy, pp 259-278 Netbiblo, Madrid, (2006)

Allan L. J. y Johnson J. A., Undergraduate attitudes toward the elderly: The role of knowledge, contact and aging anxiety, doi: 10.1080/03601270802299780, Educational Gerontology, 35(1), 1-14, (2008)

Arbuckle J. R., AMOS users guide version 21.0, Marketing Department, SPSS Incorporated, Chicago, IL, (2012)

Blanco H., Ornelas M., Tristán J. L., Cocca A., Mayorga-Vega D., López-Walle J., et al., Editor for creating and applying computerise surveys, doi: http://dx.doi.org/10.1016/j.sbspro.2013.12.105, Procedia Social and Behavioral Sciences, 106, 935-940, (2013)

Boswell S. S., Predicting trainee ageism using knowledge, anxiety, compassion, and contact with older adults, doi: 10.1080/03601277.2012.695997 Educational Gerontology, 38(11), 733-741, (2012)

Bousfield C. y Hutchison P., Contact, Anxiety, and Young People's Attitudes and Behavioral Intentions Towards the Elderly, doi: 10.1080/03601270903324362 Educational Gerontology, 36(6), 451-466, (2010)

Brzoska P. y Razum O., Validity Issues in Quantitative Migrant Health Research: The Example of IIIness Perceptions, Peter Lang International Academic Publishers, New York, NY, (2010)

Byrne B. M., Structural Equation Modeling With AMOS: Basic Concepts, Applications, and Programming, Routledge, New York, NY, (2010)

Cheung G. W. y Rensvold R. B., Evaluating goodness-of-fit indexes for testing measurement invariance, doi: 10.1207/s15328007SEM0902_5, Structural Equation Modeling, 9(2), 233-255, (2002)

Darren G. y Mallery P., IBM SPSS Statistics 21 Step by Step: A Simple Guide and Reference, Pearson, Boston, (2013)

Gelabert E., García-Esteve L., Martín-Santos R., Gutiérrez F., Torres A. y Subirà S., Psychometric properties of the Spanish version of the Frost Multidimensional Perfectionism Scale in women, Psicothema, 23(1), 133139, (2011)

Hooper D., Coughlan J. y Mullen M., Structural Equation Modelling: Guidelines for Determining Model Fit, Journal of Business Research Methods, 6(1), 53-60, (2008)

Lasher K. P. y Faulkender P. J., Measurement of Aging Anxiety: Development of the Anxiety About Aging Scale, International Journal of Aging and Human Development, 37(4), 247-259, (1993)

Montero I. y León O., Sistema de clasificación del método en los informes de investigación en Psicología, International Journal of Clinical and Health Psychology, 5, 115-127, (2005)

Nunnally J. C. y Bernstein I. H., Teoría Psicométrica, McGraw-Hill, México, (1995)

Revelle W. y Zinbarg R. E., Coefficients alpha, beta, omega and the glb: comments on Sijtsma, doi: 10.1007/s11336-008-9102-z, Psychometrika, 74(1), 145-154, (2009) 
Rivera A., Montero L. M., González A. L. y Sánchez J. J., Escala de ansiedad ante el envejecimiento de Lasher y Faulkender: Propiedades psicométricas en adultos mayores mexicanos, Salud Mental, 30(4), 5561, (2007)

Sijtsma K., On the use, the misuse, and the very limited usefulness of Cronbach's alpha, doi: 10.1007/s11336-008-9101-0, Psychometrika, 74(1), 107-120, (2009)

Thompson B., Exploratory and Confirmatory Factor Analysis. Understanding concepts and applications. , American Psychological Association, Washington, D C, (2004) 